Le Lait (1983), 63, 391-404

\title{
Influence de la flore thermorésistante du lait cru sur la qualité de conservation du lait pasteurisé exempt de recontaminations post-pasteurisation
}

\author{
par \\ R. MOURGUES*, N. DESCHAMPS* et J. AUCLAIR**
}

\section{Rés u m é}

Cinquante-deux échantillons de lait provenant de trois fermes et de trois laiteries ont été pasteurisés au laboratoire $\left(63^{\circ} \mathrm{C}, 30 \mathrm{~min}\right)$ et conservés à 6 et à $8^{\circ} \mathrm{C}$. La durée de conservation du lait pasteurisé a été mesurée en déterminant le nombre de jours pendant lequel le lait se conservait avant que le nombre de germes atteigne $30000 / \mathrm{ml}$ ou qu'un défaut de goût apparaisse.

On a cherché les corrélations existant entre, d'une part la durée de conservation du lait pasteurisé, et d'autre part la flore totale, la flore thermorésistante et les spores du lait cru. Les coefficients les plus élevés ont été trouvés pour les corrélations entre la durée de conservation à 6 et à $8^{\circ} \mathrm{C}$ et le nombre de thermorésistants (déterminés à $30^{\circ} \mathrm{C}$ ). Cela a été confirmé par l'analyse des résultats par régression multiple progressive.

Cette étude montre l'intérêt que présente le dénombrement des germes thermorésistants du lait cru destiné à la préparation du lait pasteurisé. Le nombre de thermorésistants du lait cru conditionne, en effet, non seulement la teneur en germes du lait pasteurisé mais aussi sa durée de conservation dans le cas où il n'est pas recontaminé après la pasteurisation. Les résultats obtenus confirment le rôle essentiel de la température dans la conservation du lait pasteurisé.

Mots clés :

Flore thermorésistante - Qualité de conservation - Lait pasteurisé.

* Laboratoire de Microbiologie Laitière et de Génie Alimentaire, I.N.R.A. - 78350 Jouy-en-Josas.

** Laboratoire de Biochimie et Technologie Laitières, I.N.R.A. - 78350 Jouy-enJosas. 


\section{Su m m a ry}

INFLUENCE OF THE THERMORESISTANT FLORA OF RAW MILK ON THE KEEPING QUALITY OF NON REINFECTED PASTEURIZED MILK

Fifty-two samples of milk from 3 farms and 3 dairies were laboratory pasteurized $\left(63^{\circ} \mathrm{C}, 30 \mathrm{~min}\right)$ and stored at 6 and $8^{\circ} \mathrm{C}$. The keeping quality of the pasteurized milk was determined by counting the number of days before the total bacterial count reached $30000 / \mathrm{ml}$ or before a flavour defect appeared.

Correlations have been shown between the keeping quality of the pasteurized milk and the total count, the thermoresistant count and the spore count of the raw milk. The highest coefficients were obtained for the correlations between the thermoresistant counts (determined at $30^{\circ} \mathrm{C}$ ) and the keeping quality a 6 and $8^{\circ} \mathrm{C}$. This was confirmed by the analysis of the results by progressive multiple regression.

This study shows that the thermoresistant flora of raw milk influences not only the bacterial count of the pasteurized milk but also its keeping quality in the absence of post pasteurization recontamination. The results obtained confirm the importance of the temperature in the storage of pasteurized milk.

Key words:

Thermoresistant flora - Keeping quality - Pasteurized milk.

\section{INTRODUCTION}

On sait que la qualité de conservation du lait pasteurisé est avant tout fonction des recontaminations que le lait subit après le chauffage de pasteurisation, dans les circuits de refroidissement, de stockage et de conditionnement du lait. Ce sont les bactéries psychrotrophes présentes dans ces circuits (essentiellement les Pseudomonas) qui, en se développant au cours de la conservation du lait au froid, provoquent les altérations caractéristiques du lait (défauts de goût) et limitent sa durée de conservation (Elliker et al., 1964 ; Hedrick, 1970 ; Johnson, 1979 ; Langeveld et al., 1973 ; Lausten, 1978 ; Myhr, 1972 ; Olson, 1967 ; Overcast, 1967 ; Schröder et al., 1982 ; Washam et al., 1977 ; White et Marshall, 1973).

C'est donc avant tout en réduisant les recontaminations postpasteurisation que l'on peut améliorer la qualité de conservation du lait pasteurisé. Il faut, pour cela, un circuit aussi court que possible entre le pasteurisateur et la conditionneuse et une technique de nettoyage et de stérilisation de ce circuit qui éliminent pratiquement 
toute recontamination. Rappelons qu'un seul germe psychrotrophe par litre de lait peut donner naissance à plus de 70 millions de germes par $\mathrm{ml}$ après 9 jours de conservation à $7^{\circ} \mathrm{C}$ (Overcast, 1968). C'est dire que le circuit post-pasteurisation doit être pratiquement stérile.

Nous avons cependant montré (Mourgues et Auclair, 1973) qu'en l'absence de toute recontamination post-pasteurisation, la qualité de conservation du lait pasteurisé était limitée par des bactéries provenant du lait cru, donc thermorésistantes, et capables de se développer à basse température, c'est-à-dire psychrotrophes (Bacillus, Microbacterium, etc.). En partant de 14 échantillons de lait cru de qualité bactériologique variable (51 000 à $142.10^{3}$ germes totaux par ml) nous avions obtenu un lait pasteurisé qui, après conditionnement aseptique, avait une durée de conservation à $8^{\circ} \mathrm{C}$ de 10 à 35 jours (moyenne 21 jours). Nous avions trouvé, d'autre part, une certaine relation entre la durée de conservation à $8^{\circ} \mathrm{C}$ du lait pasteurisé conditionné aseptiquement et la teneur du lait cru en germes thermorésistants.

Dans les essais qui sont décrits ici, nous avons cherché à préciser la relation pouvant exister entre la qualité de conservation du lait après pasteurisation et en l'absence de recontamination, avec la flore aérobie mésophile, la flore thermorésistante (se développant à 30-22 ou $7^{\circ} \mathrm{C}$ ) et, d'autre part, avec les germes sporulés (se développant également à $30-22$ ou $7^{\circ} \mathrm{C}$ )) présents dans le lait cru.

La qualité de conservation du lait pasteurisé était définie par le nombre de jours pendant lesquels le lait se conservait à 6 ou $8^{\circ} \mathrm{C}$ avant que le nombre de germes atteigne $30000 / \mathrm{ml}$ ou qu'un défaut de goût se manifeste dans le lait.

\section{METHODES}

\section{Echantillons de lait}

L'étude a porté sur 52 échantillons de lait cru comprenant :

- 10 échantillons de lait de ferme, prélevés dans les tanks réfrigérants de 3 fermes de la région parisienne (lait de 4 traites) ;

- 18 échantillons de lait réfrigéré (mélange de lait de 4 traites) prélevés dans les tanks de stockage d'une laiterie de la région parisienne (A) collectant un lait de bonne qualité bactériologique (contenant moins de $10^{6}$ germes totaux par $\mathrm{ml}$ ) ;

- 18 échantillons de lait réfrigéré (mélange de 4 traites) prélevés dans les tanks de stockage d'une seconde laiterie de la région parisienne (B) dont le lait était de qualité médiocre (contenant souvent plus de $10^{6}$ germes totaux par ml) ;

- 6 échantillons de lait collecté en province et transporté à une laiterie de Paris (C). Il s'agissait également de lait réfrigéré (4 traites). Les échantillons étaient prélevés dans les tanks de stockage de l'usine. 
Dans tous les cas, les échantillons (env. 3 l) étaient transportés au laboratoire dans de la glace aussitôt après le prélèvement et étaient examinés dès leur arrivée au laboratoire.

\section{Examen bactériologique du lait cru}

Les échantillons de lait cru étaient soumis aux dénombrements suivants :

- flore aérobie mésophile, sur milieu PCA (Difco 0.479) à $30^{\circ} \mathrm{C}$ pendant 3 jours ;

- spores bactériennes dénombrées après chauffage du lait à $80^{\circ} \mathrm{C}$ pendant $10 \mathrm{~min}$ et ensemencement sur milieu PCA avec incubation des boîtes de Petri à $30^{\circ} \mathrm{C}$ et $22^{\circ} \mathrm{C}$ pendant 3 jours et à $7^{\circ} \mathrm{C}$ pendant 10 jours.

\section{Pasteurisation de laboratoire}

Chaque échantillon de lait cru était réparti dans des flacons de $125 \mathrm{ml}$ bouchés à vis à raison de $100 \mathrm{ml}$ de lait par flacon. Ces flacons étaient placés dans un bain d'eau à $80^{\circ} \mathrm{C}$ jusqu'à ce que la température du lait d'un flacon témoin atteigne $63^{\circ} \mathrm{C}$. Ils étaient alors mis dans un bain d'eau réglé à $63^{\circ} \mathrm{C} \pm 1^{\circ} \mathrm{C}$ pendant $30 \mathrm{~min}$, puis refroidis successivement dans de l'eau froide et dans de l'eau glacée.

On effectuait sur l'un des flacons de lait ainsi pasteurisé un dénombrement des germes ayant résisté au traitement thermique, appelés par la suite "germes thermorésistants", sur milieu PCA avec incubation des boîtes de Petri à $30^{\circ} \mathrm{C}$ et $22^{\circ} \mathrm{C}$ pendant 3 jours et $7^{\circ} \mathrm{C}$ pendant 10 jours.

\section{Mesure de la durée de conservation du lait pasteurisé}

Les flacons de lait pasteurisé correspondant à chaque échantillon de lait cru étaient placés en nombre égal dans deux bains d'eau à $6 \pm 0,2^{\circ} \mathrm{C}$ et $8 \pm 0,2^{\circ} \mathrm{C}$.

Les lundis, mercredis et vendredis, on retirait de chacun des bains d'eau un flacon de lait qui était soumis à un dénombrement de la flore bactérienne par ensemencement sur milieu PCA avec incubation des boîtes de Petri à $30^{\circ} \mathrm{C}$ pendant 3 jours et à une dégustation destinée à détecter tout défaut de goût. Cette dégustation était effectuée sur le lait réchauffé à la température ambiante, par un groupe de trois personnes entraînées à cette détection. La conservation du lait à 6 et $8^{\circ} \mathrm{C}$ était poursuivie jusqu'à l'apparition d'un défaut de goût.

Les résultats des dénombrements et de la détermination de la durée de conservation ont été soumis à une analyse statistique de régression multiple progressive, selon un programme établi par le Laboratoire de Biométrie de l'I.N.R.A. 


\section{RESULTATS}

Le tableau 1 donne les résultats globaux des dénombrements de la flore aérobie mésophile, des germes thermorésistants (se développant à $30^{\circ} \mathrm{C}$ et $22^{\circ} \mathrm{C}$ ) et des spores (se développant à $30^{\circ} \mathrm{C}$ et $22^{\circ} \mathrm{C}$ ), obtenus pour les quatre catégories d'échantillons de lait, ainsi que les durées de conservation correspondantes, à $6^{\circ} \mathrm{C}$ et $8^{\circ} \mathrm{C}$.

Les dénombrements des germes thermorésistants et des spores se développant à $7^{\circ} \mathrm{C}$ n'ont pas été pris en compte dans ce tableau ni dans l'analyse statistique, du fait que les nombres de germes ou de spores obtenus étaient très faibles et pour les spores, souvent nuls (moins de 1 spore par ml de lait).

Dans le tableau 2 sont portés les coefficients de corrélation obtenus, pour l'ensemble des échantillons de lait, entre les résultats des dénombrements et les deux modes d'expression de la durée de conservation du lait à 6 et $8^{\circ} \mathrm{C}$ (durée de conservation avant d'atteindre 30000 germes $/ \mathrm{ml}$ et durée de conservation avant d'observer un défaut de goût). Les corrélations entre la durée de conservation et la flore aérobie mésophile d'une part, et les germes thermorésistants d'autre part, étaient presque toujours hautement significatives au seuil $\mathrm{p}=0,01$.

On voit sur ce tableau que le dénombrement des germes thermorésistants à $30^{\circ} \mathrm{C}$ donne le coefficient de corrélation le plus élevé avec la durée de conservation du lait (quelle qu'en soit le mode d'expression). Cela est confirmé par l'analyse des résultats par régression multiple progressive qui montre, en outre, que l'utilisation des autres dénombrements (flore aérobie mésophile, thermorésistants à $22^{\circ} \mathrm{C}$, sporulés à $30^{\circ} \mathrm{C}$ et $22^{\circ} \mathrm{C}$ ) n'augmente que peu la valeur du coefficient de corrélation multiple.

Les figures 1, 2, 3 et 4 montrent la répartition des échantillons de lait suivant leur durée de conservation et leur teneur en germes thermorésistants se développant à $30^{\circ} \mathrm{C}$, avec les droites de régression correspondantes.

On voit en particulier que les points correspondant aux échantillons de lait de ferme se situent très généralement au-dessus des droites de régression. Le lait de ferme se conserverait donc mieux, à nombre égal de germes thermorésistants, que le lait de mélange collecté par les laiteries.

Les droites de régression permettent de prédire, à partir du nombre de thermorésistants du lait, quelle sera en moyenne la durée de conservation à 6 et à $8^{\circ} \mathrm{C}$ du lait pasteurisé (en l'absence de recontamination). On voit, par exemple, sur le tableau 3 établi à partir des figures 1, 2, 3 et 4, qu'un lait cru contenant 10000 thermorésistants ne pourra pas se conserver, en moyenne, plus de 13 jours à $8^{\circ} \mathrm{C}$ sans défaut de goût, tandis qu'un lait contenant 100000 thermorésistants ne se conservera pas plus de 10 jours dans les mêmes conditions. 


\section{TABLEAU 1}

Résultats des dénombrements et durée de conservation de 52 échantillons de lait après pasteurisation et en l'absence de recontaminations post-pasteurisation

\begin{tabular}{|c|c|c|c|c|c|c|}
\hline \multicolumn{3}{|c|}{ Nombre d'échantillons } & Lait de ferme & Laiterie A & Laiterie B & Laiterie C \\
\hline $\begin{array}{l}\text { Germes aérobies mésophiles } \\
\text { (par ml) }\end{array}$ & \multicolumn{2}{|c|}{$\begin{array}{l}\text { Moyenne logarithmique } \\
\text { Valeurs extrêmes }\end{array}$} & $\begin{array}{c}91000 \\
22000-1.106\end{array}$ & $\begin{array}{c}142000 \\
42000-750000\end{array}$ & $\begin{array}{c}1,6.10^{6} \\
75000-20.10^{6}\end{array}$ & $\begin{array}{c}2,5.106 \\
375000-8,5.10^{6}\end{array}$ \\
\hline $\begin{array}{l}\text { Thermorésistants à } 30^{\circ} \mathrm{C} \\
\text { (par ml) }\end{array}$ & \multicolumn{2}{|c|}{$\begin{array}{l}\text { Moyenne logarithmique } \\
\text { Valeurs extrêmes }\end{array}$} & $\begin{array}{c}2000 \\
76-390000\end{array}$ & $\begin{array}{c}1500 \\
750-3600\end{array}$ & $\begin{array}{c}23000 \\
7600-256000\end{array}$ & $\begin{array}{c}31300 \\
2000-61000\end{array}$ \\
\hline $\begin{array}{l}\text { Thermorésistants à } 22^{\circ} \mathrm{C} \\
\text { (par ml) }\end{array}$ & \multicolumn{2}{|c|}{$\begin{array}{l}\text { Moyenne logarithmique } \\
\text { Valeurs extrêmes }\end{array}$} & $\begin{array}{l}400 \\
6-100000\end{array}$ & $\begin{array}{l}620 \\
10-2600\end{array}$ & $\begin{array}{l}1300 \\
200-200000\end{array}$ & $\begin{aligned} & 3800 \\
73 & -42000\end{aligned}$ \\
\hline Spores à $30^{\circ} \mathrm{C}$ (par ml) & \multicolumn{2}{|c|}{$\begin{array}{l}\text { Moyenne logarithmique } \\
\text { Valeurs extrêmes }\end{array}$} & $\begin{array}{c}110 \\
8-2800\end{array}$ & $\begin{array}{c}180 \\
64-940\end{array}$ & $\begin{array}{c}190 \\
51-1600\end{array}$ & $\begin{array}{l}340 \\
16-5700\end{array}$ \\
\hline Spores à $22^{\circ} \mathrm{C}$ (par ml) & \multicolumn{2}{|c|}{$\begin{array}{l}\text { Moyenne logarithmique } \\
\text { Valeurs extrêmes }\end{array}$} & $\begin{array}{l}17 \\
4-1400\end{array}$ & $27-660$ & $18 \stackrel{65}{-550}$ & $\begin{array}{c}76 \\
7-3700\end{array}$ \\
\hline \multirow{2}{*}{$\begin{array}{l}\text { Durée de conservation avant } \\
\text { d'atteindre } 30000 \text { germes } \\
\text { par ml (j) }\end{array}$} & & $\begin{array}{l}\text { Moyenne logarithm. } \\
\text { Valeurs extrêmes }\end{array}$ & $\begin{array}{c}26,4 \\
4-37\end{array}$ & $\begin{array}{l}12,8 \\
6-21\end{array}$ & $\begin{array}{l}8,5 \\
3-16\end{array}$ & $\begin{array}{c}9,7 \\
3-14\end{array}$ \\
\hline & $8^{\circ} \mathrm{C}$ & $\begin{array}{l}\text { Moyenne logarithm. } \\
\text { Valeurs extrêmes }\end{array}$ & $2-22$ & $\begin{array}{c}9,0 \\
5-10\end{array}$ & $2-9$ & $\begin{array}{c}5,8 \\
2-7\end{array}$ \\
\hline \multirow{2}{*}{$\begin{array}{l}\text { Durée de conservation avant } \\
\text { défaut de goût (j) }\end{array}$} & $6^{\circ} \mathrm{C}$ & $\begin{array}{l}\text { Moyenne logarithm. } \\
\text { Valeurs extrêmes }\end{array}$ & $\begin{array}{c}29,0 \\
19-37\end{array}$ & $\begin{array}{c}24,4 \\
16-33\end{array}$ & $\begin{array}{c}19,8 \\
10-27\end{array}$ & $\begin{array}{c}22,7 \\
19-28\end{array}$ \\
\hline & $8^{\circ} \mathrm{C}$ & $\begin{array}{l}\text { Moyenne logarithm. } \\
\text { Valeurs extrêmes }\end{array}$ & $\begin{array}{c}18,6 \\
9-23\end{array}$ & $\begin{array}{c}14,9 \\
12 \cdot 17\end{array}$ & $\begin{array}{l}12,0 \\
7-16\end{array}$ & $\begin{array}{c}12,6 \\
11-16\end{array}$ \\
\hline
\end{tabular}


TABLEAU 2

Corrélation entre durée de conservation du lait à 6 et $8^{\circ} \mathrm{C}$ et différents dénombrements

\begin{tabular}{|c|c|c|c|c|c|c|}
\hline & & $\begin{array}{l}\text { Germes aérobies } \\
\text { mésophiles }\end{array}$ & $\begin{array}{c}\text { Thermorésistants } \\
\text { à } 30^{\circ} \mathrm{C}\end{array}$ & $\begin{array}{c}\text { Thermorésistants } \\
\text { à } 22^{\circ} \mathrm{C}\end{array}$ & Spores à $30^{\circ} \mathrm{C}$ & Spores à $22^{\circ} \mathrm{C}$ \\
\hline \multirow[t]{2}{*}{$\begin{array}{l}\text { Durée de conservation } \\
\text { avant d'atteindre } 30000 \\
\text { germes/ml (j) }\end{array}$} & $6^{\circ} \mathrm{C}$ & $\begin{array}{l}-0,65^{* *} \\
(43)\end{array}$ & $\begin{array}{l}-0,68 * * \\
(43)\end{array}$ & $\begin{array}{c}-0,63^{* *} \\
(43)\end{array}$ & $\begin{array}{c}-0,50 * * \\
(43)\end{array}$ & $\begin{array}{l}-0,46^{* *} \\
(43)\end{array}$ \\
\hline & $8^{\circ} \mathrm{C}$ & $\begin{array}{l}-0,67^{* *} \\
(45)\end{array}$ & $\begin{array}{l}-0,79 * * \\
(45)\end{array}$ & $\begin{array}{l}-0,75^{* *} \\
(45)\end{array}$ & $\begin{array}{l}-0,53 * * \\
(45)\end{array}$ & $\begin{array}{l}-0,53^{* *} \\
(45)\end{array}$ \\
\hline \multirow[t]{2}{*}{$\begin{array}{l}\text { Durée de conservation } \\
\text { avant défaut de goût }(j)\end{array}$} & $6^{\circ} \mathrm{C}$ & $\begin{array}{c}-0,62 * * \\
(43)\end{array}$ & $\begin{array}{c}-0,65^{* *} \\
(43)\end{array}$ & $\begin{array}{c}-0,53^{* *} \\
(43)\end{array}$ & $\begin{array}{c}-0,34^{*} \\
(43)\end{array}$ & $\begin{array}{c}-0,31^{*} \\
(43)\end{array}$ \\
\hline & $8^{\circ} \mathrm{C}$ & $\begin{array}{c}-0,72^{* *} \\
(43)\end{array}$ & $\begin{array}{c}-0,76^{* *} \\
(43)\end{array}$ & $\begin{array}{c}-0,65^{* *} \\
(43)\end{array}$ & $\begin{array}{c}-0,50 * * \\
(43)\end{array}$ & $\begin{array}{c}-0,43^{* *} \\
(43)\end{array}$ \\
\hline
\end{tabular}

Entre parenthèses, nombre de données $\mathrm{N}$.

* Corrélation significative. Seuil p $=0,05$.

** Corrélation hautement significative. Seuil p $=0,01$. 


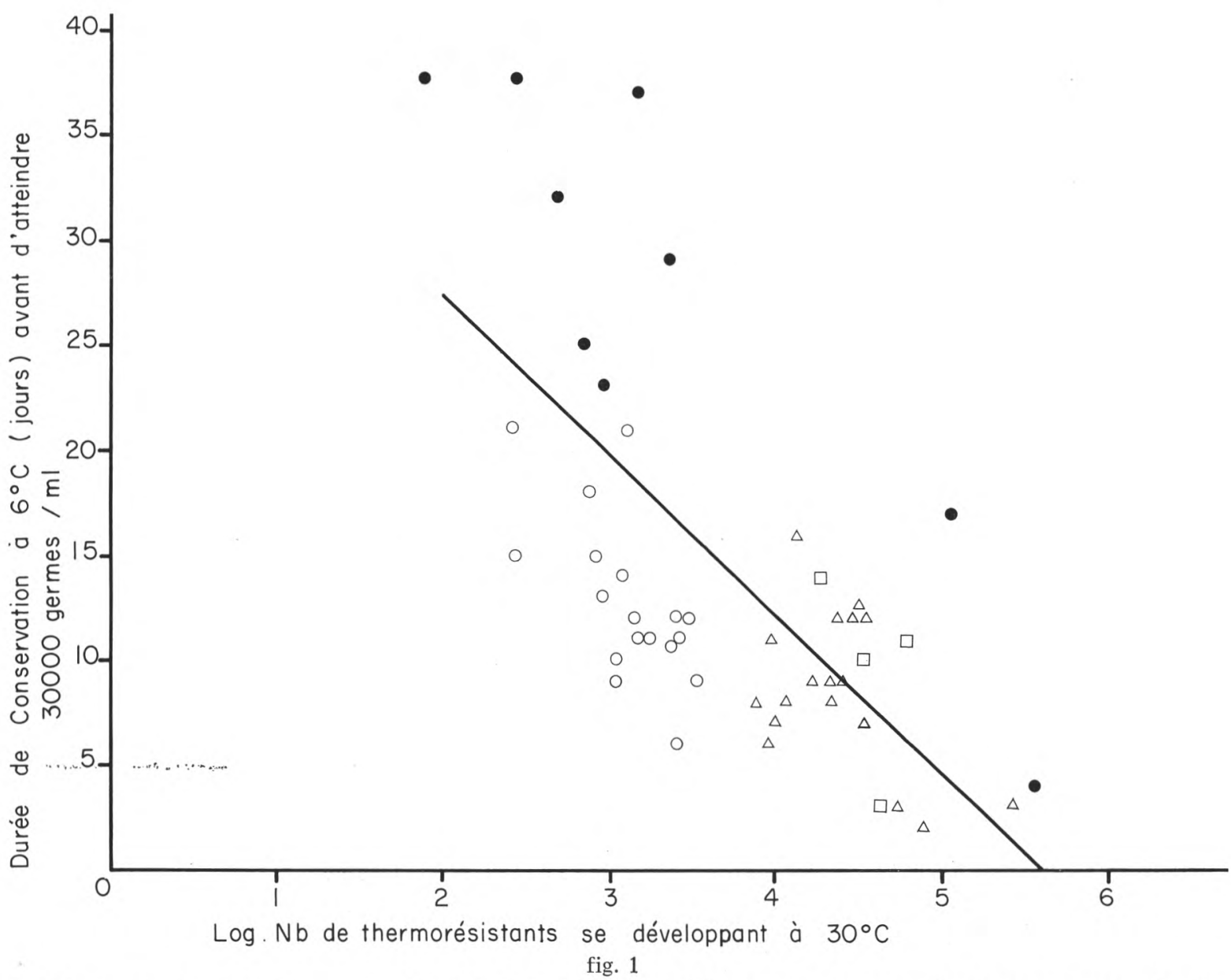

Relation entre la durée de conservation à $6^{\circ} C$ du lait pasteurisé exempt de recontaminations post-pasteurisation et le nombre de germes thermorésistants dénombrés à $30^{\circ} \mathrm{C}$ (durée de conservation exprimée par le nombre de jours avant que le nombre de germes du lait atteigne 30000 par ml).

- Lait de ferme, O Laiterie A, $\triangle$ Laiterie B, $\square$ Laiterie C.

Relation between the shelf life at $6^{\circ} \mathrm{C}$ of post pasteurization contamination free pasteurized milk and the number of thermodurics counted at $30^{\circ} \mathrm{C}$ (shelf life expressed by the number of days before the bacterial count reached 30000 per $\mathrm{ml}$ ).

- Farm milk, O Dairy $A, \triangle$ Dairy B, 口 Dairy $C$. 


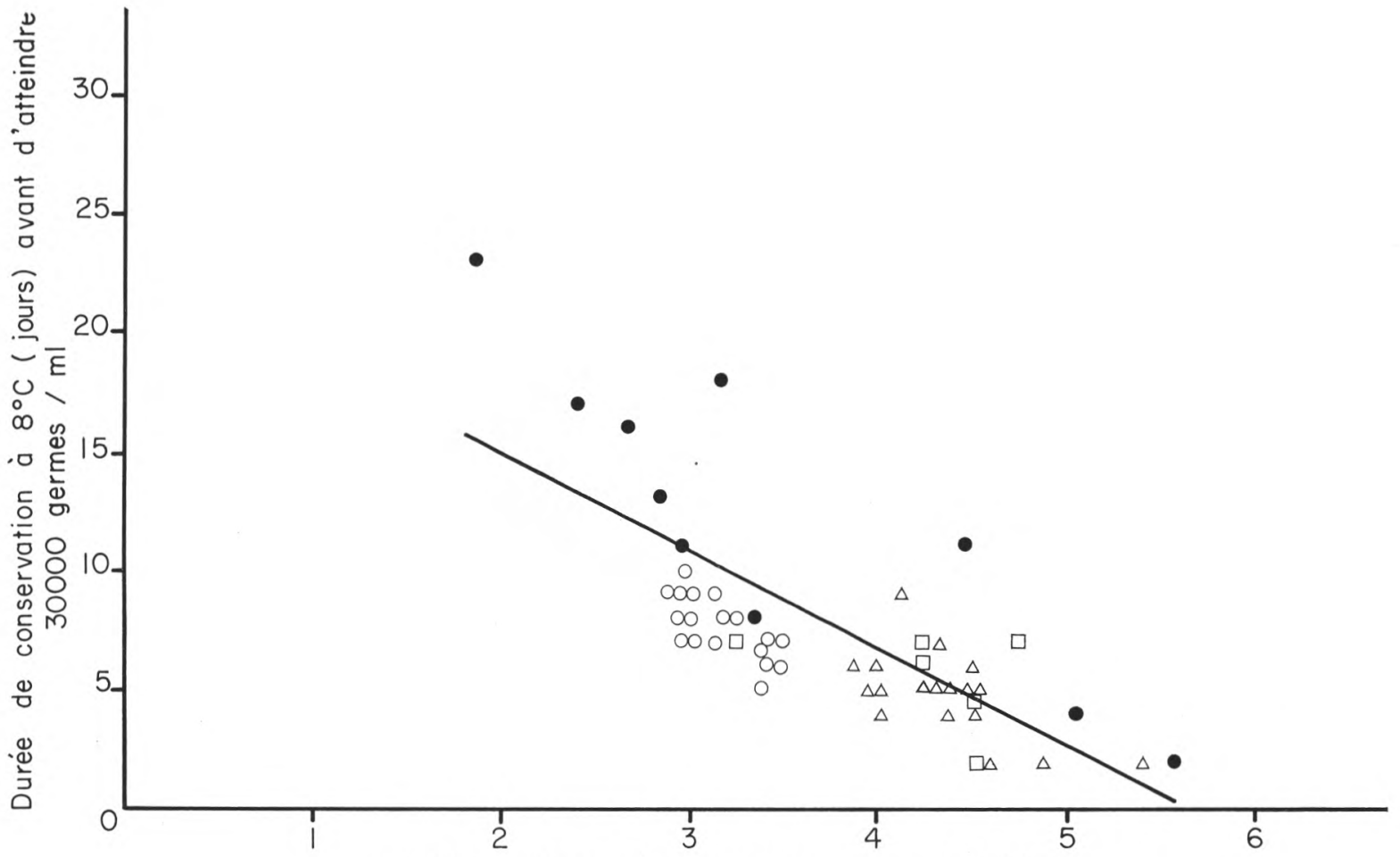

Relation entre la durée de conservation à $8^{\circ} \mathrm{C}$ du lait pasteurisé exempt de recontaminations post-pasteurisation et le nombre de germes thermorésistants dénombrés à $30^{\circ} \mathrm{C}$ (durée de conservation exprimée par le nombre de jours avant que le nombre de germes du lait atteigne 30000 par ml).

- Lait de ferme, O Laiterie A, $\triangle$ Laiterie B, $\square$ Laiterie C.

Relation between the shelf life at $8^{\circ} \mathrm{C}$ of post pasteurization contamination free pasteurized milk and the number of thermodurics counted at $30^{\circ} \mathrm{C}$ (shelf life expressed by the number of days before the bacterial count reached 30000 per $\mathrm{ml}$ ).

- Farm milk, O Dairy $A, \triangle$ Dairy $B$, 口 Dairy $C$. 


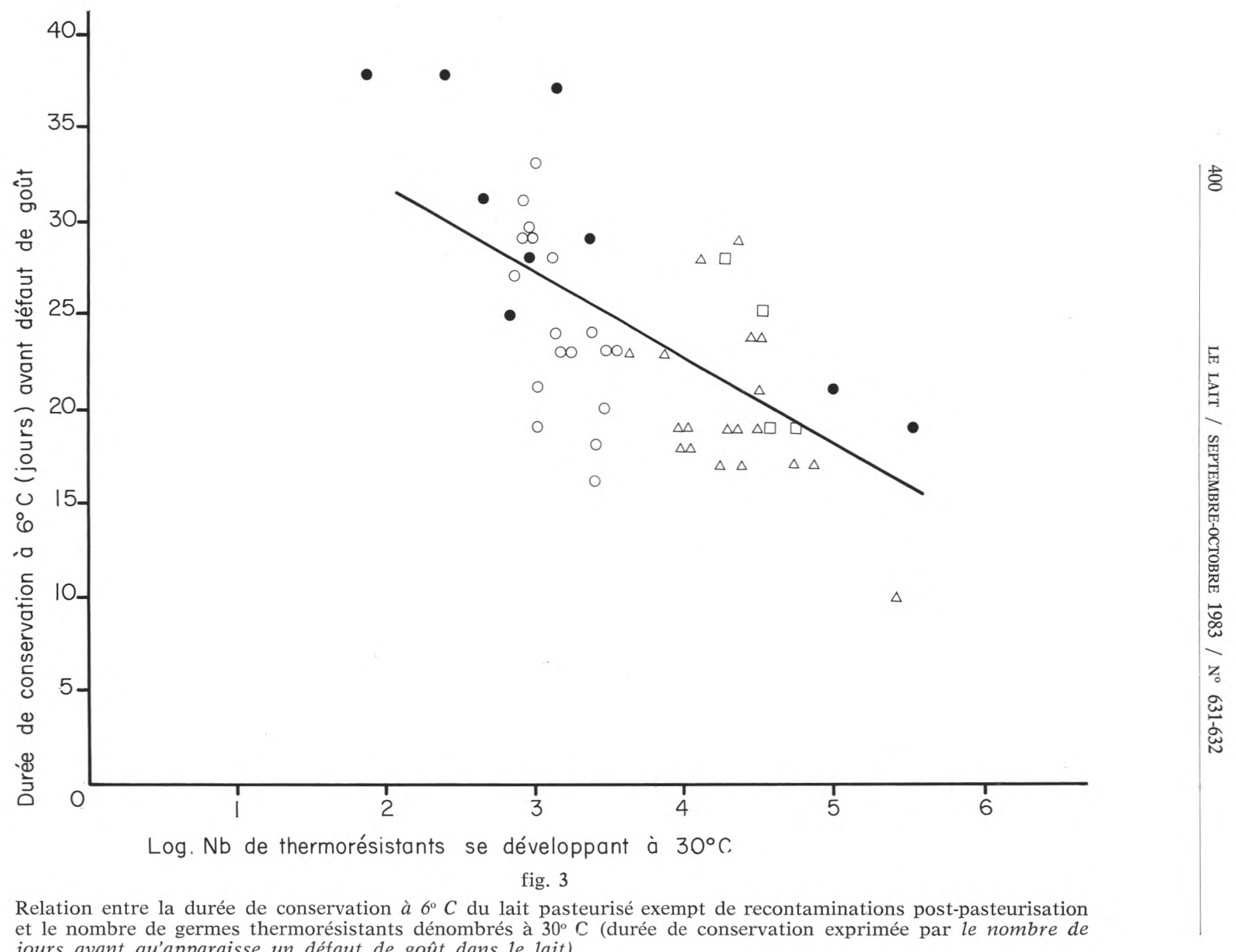

et le nombre de germes thermorésistants dénombrés à $30^{\circ} \mathrm{C}$ (durée de conservation exprimée par le nombre de jours avant qu'apparaisse un défaut de goût dans le lait).

- Lait de ferme, $O$ Laiterie A, $\triangle$ Laiterie B, $\square$ Laiterie C.

Relation between the shelf life at $6^{\circ} \mathrm{C}$ of post pasteurization contamination free pasteurized milk and the number of thermodurics counted at $30^{\circ} \mathrm{C}$ (shelf life expressed by the number of days before a flavor defect appeared).

- Farm milk, ○ Dairy $A, \triangle$ Dairy $B$, $\square$ Dairy $C$. 


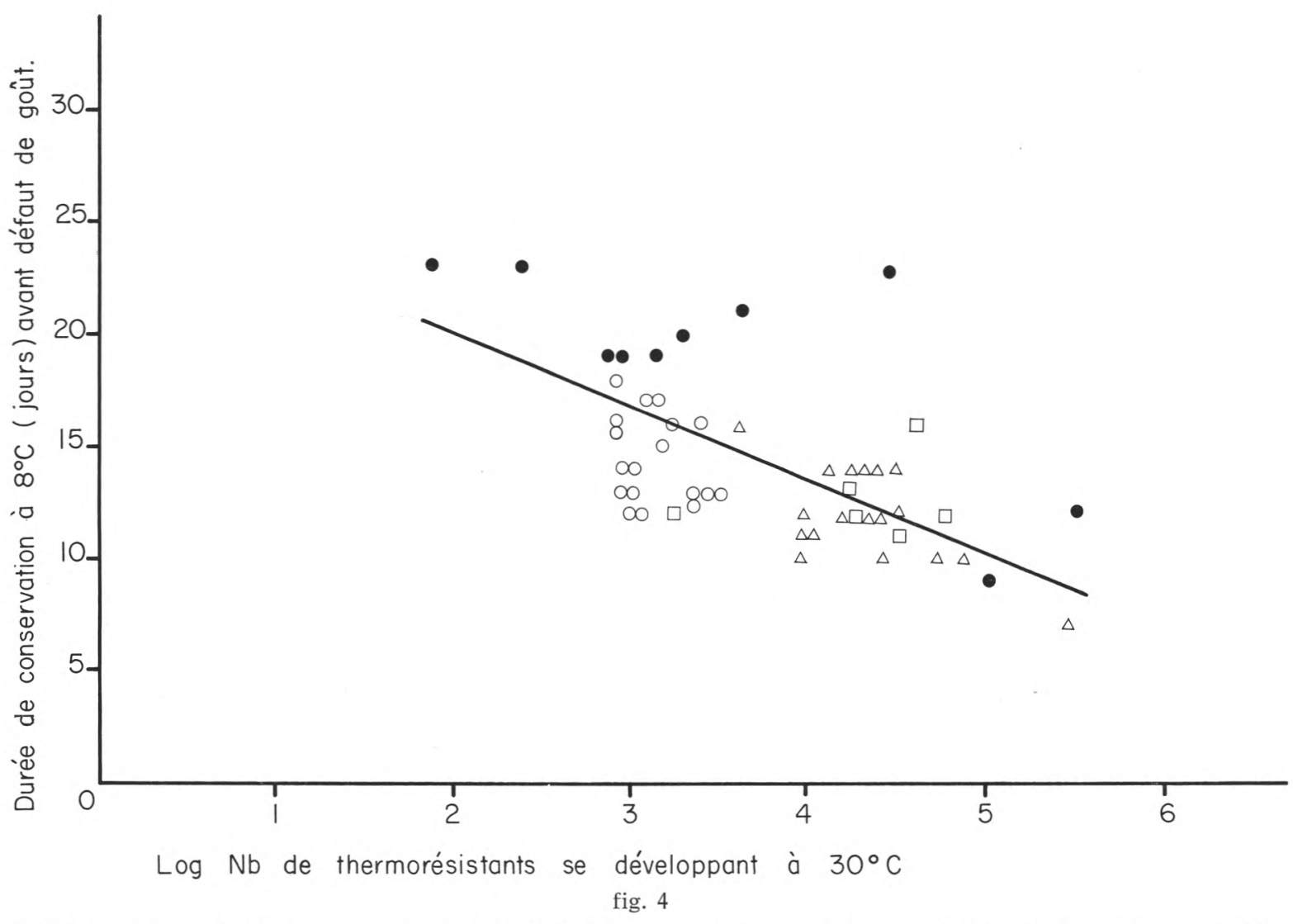

et le nombre de germes thermorésistants dénombrés à $30^{\circ} \mathrm{C}$ (durée de conservation exprimée par le nombre de jours avant qu'apparaisse un défaut de goût dans le lait).

- Lait de ferme, O Laiterie A, $\triangle$ Laiterie B, $\square$ Laiterie C.

(hetween the shelf life at $8^{\circ} \mathrm{C}$ of post pasteurization contamination free pasteurized milk and the number of thermodurics counted at $30^{\circ} \mathrm{C}$ (shelf life expressed by the number of days before a flavor defect appeared). - Farm milk, O Dairy A, $\triangle$ Dairy $B$, Dairy $C$. 
TABLEAU 3

Relation entre la flore thermorésistante du lait cru et la durée moyenne de conservation du lait pasteurisé (en jours)

\begin{tabular}{|c|c|c|c|c|}
\hline \multirow{3}{*}{$\begin{array}{c}\text { Nombre } \\
\text { de } \\
\text { thermo- } \\
\text { résistants }\end{array}$} & \multicolumn{4}{|c|}{ Durée de conservation à } \\
\hline & \multicolumn{2}{|c|}{$6^{\circ} \mathrm{C}$} & \multicolumn{2}{|c|}{$8^{\circ} \mathrm{C}$} \\
\hline & $\begin{array}{c}\text { pour } \\
\text { atteindre } 30000 \\
\text { germes } / \mathrm{ml}\end{array}$ & $\begin{array}{l}\text { avant } \\
\text { défaut de } \\
\text { goût }\end{array}$ & $\begin{array}{c}\text { pour } \\
\text { atteindre } 30000 \\
\text { germes } / \mathrm{ml}\end{array}$ & $\begin{array}{l}\text { avant } \\
\text { défaut de } \\
\text { goût }\end{array}$ \\
\hline 1000 & 20 & 27 & 11 & 17 \\
\hline 10000 & 12 & 23 & 7 & 13 \\
\hline 100000 & $4^{*}$ & 18 & $3 *$ & 10 \\
\hline
\end{tabular}

\section{DISCUSSION}

L'altération du lait pasteurisé exempt de recontaminations postpasteurisation est due essentiellement au développement des bactéries sporulées aérobies du genre Bacillus et en particulier : B. cereus (Coghill et Juffs, 1979 ; Credit et al., 1972 ; Grosskopf et Harper, 1974 ; Johnston et Bruce, 1982 ; Langeveld et al., 1973 ; Overcast et Atmaran, 1974 ; Shehata et Collins, 1971 ; Washam et al., 1977). On aurait donc pu s'attendre à ce que la durée de conservation du lait pasteurisé soit reliée avant tout au nombre de germes sporulés du lait cru (dénombrés à 7,22 ou $30^{\circ} \mathrm{C}$ ). Le fait qu'il n'en est pas ainsi vient sans doute de ce que les nombres de spores trouvées dans le lait cru sont relativement faibles et, dans ces conditions, la nature des germes en question est probablement plus importante que leur nombre vis-à-vis de la qualité de conservation du lait.

La corrélation entre durée de conservation du lait et nombre de germes thermorésistants (déterminés à $30^{\circ} \mathrm{C}$ ) est donc intéressante. Elle s'explique sans doute en partie par le fait que le traitement thermique appliqué au lait était le même pour la détermination du nombre de thermorésistants et pour la mesure de la durée de conservation du lait. La corrélation aurait sans doute été moins bonne si la conservation avait été mesurée dans du lait pasteurisé à température 
plus élevée comme c'est le cas de la plus grande partie du lait pasteurisé en France.

Cependant, la corrélation hautement significative trouvée dans nos essais entre la qualité de conservation du lait et le nombre de thermorésistants a plusieurs conséquences :

Comme indiqué précédemment, il est possible de prévoir, suivant le nombre de thermorésistants du lait cru la durée maximale de conservation du lait pasteurisé, c'est-à-dire la durée qui pourrait être atteinte si toute recontamination post-pasteurisation était supprimée grâce à des circuits pratiquement aseptiques. Cela veut dire aussi que si le nombre de thermorésistants du lait cru atteint un certain niveau, on ne peut pas espérer obtenir une longue durée de conservation, quels que soient les soins d'hygiène pris au niveau des circuits post-pasteurisation.

Connaissant l'influence importante des germes thermorésistants du lait cru sur la qualité de conservation du lait pasteurisé, on conçoit l'importance qu'il y a pour le transformateur à utiliser du lait cru aussi peu chargé que possible en thermorésistants, et la nécessité pour cela d'engager une action systématique de détection des laits de producteurs riches en thermorésistants et d'information auprès des producteurs pour faire baisser le nombre de thermorésistants de leur lait. Eventuellement, les laits de producteurs constamment très chargés en germes thermorésistants devraient être refusés.

Ainsi, la réduction du nombre de germes thermorésistants du lait pasteurisé n'est pas seulement nécessaire pour respecter une obligation réglementaire (moins de 30000 germes $/ \mathrm{ml}$ ) mais aussi pour améliorer la qualité de conservation du lait.

L'observation suivant laquelle le lait de ferme semble se conserver mieux, à nombre égal de thermorésistants, que le lait de mélange reçu par les laiteries est également à noter. Cette différence pourrait s'expliquer par le fait que le lait de ferme, n'ayant subi avant son traitement thermique qu'un développement microbien limité, est un milieu de culture peu favorable aux bactéries qui se développent dans ce lait après pasteurisation au cours de sa conservation au froid. Par contre le lait de mélange qui, lui, a été le siège (en particulier au cours du transport du lait) d'un développement plus ou moins important de germes psychrotrophes, est un milieu de culture plus favorable et de ce fait se conserve moins bien. On retrouve ici, avec le lait de ferme, des durées de conservation du lait pasteurisé élevées, comparables à celles qui avaient été observées dans une étude précédente, réalisée sur des échantillons de lait de ferme (Mourgues et Auclair, 1973).

Nos résultats confirment enfin le rôle primordial de la température dans la durée de conservation du lait pasteurisé comme on le voit sur le tableau 3, l'abaissement de la température de conservation du lait de 8 à $6^{\circ} \mathrm{C}$ permet d'augmenter de 8 à 10 jours la durée de conservation avant apparition d'un défaut de goût. 


\section{Bibliographie}

COGHILl (D.) and JufFs (H.S.) (1979), - Incidence of psychrotrophic spore forming bacteria in pasteurized milk and cream products and effect of temperature on their growth. Australian Journal of Dairy Technology, 34, 150-153.

Credit (C.), Heneman (R.), Herwood (R.) and Westhoff (D.) (1972). - Identification of bacteria isolated from pasteurized milk following refrigerated storage. Journal of milk and Food Technology, 35, 708-709.

GrossKopf (P. F.) and HARPER (W. J.) (1974). - Isolation and identification of psychrotrophic spore-formers in the milk. Milchwissenschaft, 29, 467-470.

HEDRICK (T. I.) (1970). - How to keep milk fresh for 3 weeks. American Dairy Review, 32 (4), 56-59.

Elliker (P. R.), Sing (E. L.), Christensen (L. J.) and Sandine (W. E.) (1964). Psychrophilic bacteria and keeping quality of pasteurized dairy products. Journal of Milk and Food Technology, 27, 69-75.

Johnson (C. L.) (1979). - How to get twenty days shelf-life. American Dairy Review, 41 (7), 24-46.

Johnston (D. W.) and BRUCE (J.) (1982). - Incidence of thermoduric psychrotrophs in milk produced in the West of Scotland. Journal of Applied Bacteriology, 52, 333-337.

Langeveld (L. P. M.), Cuperus (F.) and Stadhouders (J.) (1973). - Bacteriological aspects of the keeping quality at $5^{\circ} \mathrm{C}$ of reinfected and non reinfected milk. Netherlands Milk and Dairy Journal, 27, 54-65.

LAUSTEN (K.) (1978). - Development of aerobic psychrotrophs in whole and skim milk. Dansk Veterinaertidsskrift, 61, 1098-1109.

Mourgues (R.) et Auclair (J.) (1973). - Durée de conservation à $4^{\circ} \mathrm{C}$ et à $8^{\circ} \mathrm{C}$ du lait pasteurisé conditionné aseptiquement. Le Lait, 53, 481-490.

Myhr (A. N.) (1972) . - Better shelf life is possible only if plant sanitation gets proper attention. Modern Dairy, 51 (1), 8-9.

OLSON (H.C.) (1967), - Relationship between bacterial counts and shelf life of milk (abstract). Journal of Dairy Science, 50, 607.

Overcast (W. W.) (1967). - Extending shelf life by controlling psychrophiles. American Dairy Review, 29 (9), 42-48.

Overcast (W. W.) (1968). - Psychrophilic microorganisms and keeping quality of milk and its products. Journal of Dairy Science, 51, 1336-1338.

Overcast (W. W.) and Atmaram (K.) (1974), - The role of Bacillus cereus in sweet curdling of fluid milk. Journal of Milk and Food Technology, 37, 233-236.

SchröDER (M. J. A.), Cousins (C. M.) and Mc KinnoN (C. H.) (1982). - Effect of psychrotrophic post-pasteurization contamination on the keeping quality at 11 and $5^{\circ} \mathrm{C}$ of HTST-pasteurized milk in the U.K. Journal of Dairy Research, 49, 619-630.

Shehata (T. E.) and Collins (E. B.) (1971). - Isolation and identification of psychrotrophic species of Bacillus from milk. Journal of Applied Microbio$\log y, 21,466-469$.

Washam (C. J.), Olson (H. C.) and Vedamuthu (E. R.) (1977). - Heat resistant psychrotrophic bacteria isolated from pasteurized milk. Journal of Food Protection, 40, 101-108.

White (C. H.) and Marshall (R. T.) (1973). - Reduction of shelf life of dairy products by a heat stable protease from P. fluorescens. Journal of Dairy Science, 56, 849-853. 